\title{
LA CONVIVENCIA ESCOLAR: UNA ARISTA DE PAZ EN MEDIO DEL POSCONFLICTO
}

\author{
Karen Estefanni Pérez Álvarez* \\ Flor Liliana Castellanos Bothia** \\ Recibido: Marzo 18 de 2019 \\ Aprobado: Abril 23 de 2019
}

\section{RESUMEN:}

El presente texto tiene como objetivo describir la importancia de implementar estrategias de gestión pacífica de conflictos en las aulas, con el fin de prevenir y mitigar la violencia en las instituciones escolares y generar escenarios de inclusión y convivencia, utilizando el Derecho como herramienta que permite, desde el enfoque en derechos humanos, transformar la realidad de las instituciones escolares. Para ello se sugiere la implementación de propuestas por medio de las cuales los niños, niñas y adolescentes serán agentes de paz en esta nueva era que le espera a Colombia: el posconflicto, un escenario que involucra la participación de la sociedad, pero, en especial, y como pilar de nuestro planteamiento, involucra la participación de las instituciones educativas. La propuesta tiene un componente de arte para sensibilizar y mediar la comunicación

Citar este trabajo como: Pérez Álvarez, K \& Castellanos Bothia, F. (2019). La convivencia escolar: una arista de paz en medio del posconflicto. Temas Socio-Jurídicos, 38(76), pp. 155-166. https://doi.org/ 10.29375/01208578.3582

* Estudiante de derecho de la Universidad Autónoma de Bucaramanga, integrante de la línea de Género y Derecho de la clínica Jurídica de Derechos Humanos e Interés Público. Vinculada al semillero Género y Diversidad. Orcid: 0000-0002-5688-0233. Correo electrónico: kperez244@unab.edu.co

** Estudiante de derecho de la Universidad Autónoma de Bucaramanga, integrante de la línea de Género y Derecho de la clínica Jurídica de Derechos Humanos e Interés Público. Vinculada al semillero Género y Diversidad. Orcid: 0000-0003-0800-2425F. Correo electrónico: castellanos547@unab.edu.co 
con niños, niñas y adolescentes, y abrir la mente a nuevas estrategias e iniciativas para la construcción de paz desde las aulas.

Palabras Clave: Paz escolar; Instituciones educativas; violencia escolar; derechos humanos.

\title{
THE SCHOOL COEXISTENCE: AN ASPECT OF PEACE IN THE MIDDLE OF THE POST-CONFLICT
}

\begin{abstract}
:
The purpose of this document is to describe the importance of implementing strategies to peacefully manage conflicts in the classrooms, with the aim of preventing and mitigating violence in educational institutions and generating spaces for inclusion and coexistence, utilizing the Law as a tool to transform the reality at educational institutions from a Human Rights approach. This suggests the implementation of proposals through which children and adolescents become agents of peace in this new era that awaits Colombia: the post-conflict, a scenario that involves society participation, but in particular, and as a pillar of our exposition, requires the participation of the educational institutions. The proposal has an art component to raise awareness, to mediate communication with the children and adolescents, and to open the mind to new strategies and initiatives to construct peace from within the classrooms.
\end{abstract}

Keywords: School peace; educational institutions; school violence; human rights.

\section{O CONVÍVIO ESCOLAR: UMA ARESTA DE PAZ NO MEIO DO PÓS-CONFLITO}

\section{RESUMO:}

O presente texto tem como objetivo descrever a importância da implementação de estratégias para a gestão pacífica de conflitos na sala de aula, a fim de prevenir e reduzir a violência nas instituições educacionais e gerar cenários de inclusão e convivência, utilizando o Direito como ferramenta que permite, a partir do foco em direitos humanos, transformar a realidade das instituições educacionais. Por tanto, sugere-se a implementação de propostas por meio das quais crianças e adolescentes serão agentes de paz nesta nova era que aguarda a Colômbia: o pós-conflito, um cenário que envolve a participação da sociedade, mas especialmente e, como pilar de nossa abordagem, envolve a participação das instituições educacionais. A proposta tem um componente artístico para sensibilizar 
e mediar a comunicação com crianças e adolescentes e abrir a mente para novas estratégias e iniciativas para a construção de paz na sala de aula.

Palavras-chave: Paz escolar; Instituições educacionais; violência escolar; direitos humanos.

\section{INTRODUCCIÓN}

En el 2014 Colombia se conmovió por un lamentable suceso en Bogotá: los medios de comunicación dieron a conocer la muerte de un joven de 17 años; su nombre era Sergio Urrego Reyes. Las circunstancias de su muerte giran en torno a una situación de acoso escolar por razones de orientación sexual que hicieron que él mismo terminara con su vida ${ }^{1}$.

A la luz de ese hecho y tras la invitación de Colombia Diversa para realizar una intervención en la revisión de la tutela del caso de Sergio Urrego por parte de la Corte Constitucional, nace un proyecto de investigación al interior del Semillero Género y Derecho ${ }^{2}$ sobre acoso escolar por razones de orientación sexual, en aras de debatir la necesidad de proteger y tutelar derechos fundamentales que se ven amenazados o vulnerados en situaciones de acoso escolar - también llamado bullying o matoneo- dentro de las instituciones escolares.

De esta manera, la Clínica Jurídica comienza una línea de trabajo con investigación formativa y con un enfoque en género, a fin de brindar asesoría legal a niños, niñas y adolescentes en el entorno escolar, en situaciones donde estén siendo afectados o amenazados por discriminación producto de los estereotipos de género.

¿Qué explica las situaciones de conflicto en la escuela?, ¿Por qué hablar de paz en la escuela? Durante muchos años Colombia ha padecido actos violentos y las secuelas de la guerra, reclutamiento forzado de menores de edad, desplazamientos, enfrentamientos -fuego cruzado-, narcotráfico, secuestros, extorsiones, entre otras vulneraciones, que modificaron el curso de vida de los colombianos, al ser víctimas directas o por sentir el dolor de un país transformado por la misma guerra.

En el marco del proceso de paz y de la firma de los acuerdos, se empezó a replantear la visión frente al conflicto en Colombia. El comienzo del fin del conflicto durante años se percibió como una utopía. Sin embargo, no fue un deseo inalcanzable; por el contrario, siguiendo a Botero Uribe (2015),

1 La investigación penal ya ha llevado a la condena de los funcionarios de la institución escolar de Sergio. Ver: https://www.elespectador.com/noticias/bogota/segunda-condenaen-caso-sergio-urrego-psicologa-fue-sentenciada-35-meses-de-prision-articulo-711455

2 Semillero adscrito al centro de investigaciones socio jurídicas de la Facultad de Ciencias Jurídicas y Políticas de la Universidad Autónoma de Bucaramanga. 
la utopía se ve como "la posibilidad de formular propuestas abiertas que pueden reformularse, adaptarse, mejorarse e incluso sustituirse cuando no sirvan" (p.31). Así, los diálogos de paz y la posterior firma de los acuerdos se pueden ver como la posibilidad de construir una mejor Colombia.

La guerra y la paz no son un asunto solo de armas y de grandes acuerdos. La reflexión en torno a cómo atender la problemática de discriminación trascendió la esfera de armonizar las aulas y generar espacios de respeto, e inclusión, para pasar a ser parte también de ese escenario de posconflicto, que implica crear un espacio de sana convivencia escolar y, de esta manera, que las instituciones escolares estén preparadas para la inclusión de niños y niñas que fueron víctimas y excombatientes del conflicto armado. Para ello se establecen propuestas que nos permiten formar niños, niñas y adolescentes en nociones de derechos humanos, de construcción de paz, utilizando elementos del arte como lenguaje y herramienta para crear iniciativas de paz.

En lo que sigue, se describirá la propuesta metodológica, así como los resultados parciales del desarrollo y aplicación de la propuesta.

\section{REFERENTES TEÓRICOS Y JURÍDICOS}

En este apartado se describirán algunos elementos teóricos y normativos con el fin de analizar el problema de la convivencia escolar, el conflicto y la forma de construir escenarios de paz en la escuela.

\section{-Violencia - Teoría del conflicto de Galtung ${ }^{3}$}

La historia de Colombia ha sido contada en medio de difíciles situaciones, conflictos sociales y culturales que han hecho vulnerable a la comunidad y la han puesto en condiciones que alteran la estabilidad de la vida de las personas. En pocas palabras, repasar la historia de nuestro país es notar que en algunos o en varios momentos se ha manifestado la violencia; pero, ¿qué tipo de violencia? Algunos relacionarán la violencia de acuerdo con la época en la que creen haberla padecido, por ejemplo, al vincularla con los diferentes asesinatos a periodistas y políticos, y con atentados realizados contra empresas públicas; otros tantos traerán la imagen de una Colombia afectada por el narcotráfico y las bandas criminales; otros, verán la violencia desde el conflicto armado que ha padecido el país por años y que ha dejado múltiples muertes, secuestros, niños reclutados, y familias desintegradas y desplazadas. Una gran parte de la población, siente la violencia al no contar

3 García, V. (s.f). Johan Galtung.Calderón, P. (2009). Teoría de conflictos de Johan Galtung. Revista paz y conflictos ; más desarrollado en Galtung, Johan (1980), The Basic Needs Approach, en Katrin Lederer, David Antal y Johan Galtung (Eds), Human Needs: A Contribution to the Current Debate, Cambridge (Massachusetts), Oelgeschla-ger, Gunn \& Hain; Koningstein, Anton Hain. 
con una buena atención médica, no poder caminar por las calles con seguridad, o no contar con estudio ni con ayuda para conseguirlo.

Pareciera que la violencia estuviese conformada por muchas aristas desde las cuales las personas se han enfrentado a ella; no obstante, se debe mencionar una tan visible como las demás pero que quizá es pasada por alto; esta es la que tiene que ver con actos, los actos de violencia: un simple hecho o manifestación en la vida cotidiana que puede tener contenido violento. Para explicar un poco sobre esta arista, hablamos la escuela como escenario para evidenciar diferentes manifestaciones que constituyen violencia, por ejemplo, en los salones de clase, donde las expresiones mal usadas, los insultos, el exceso de fuerza en un juego, las burlas por las características de los compañeros, la discriminación por razones de género, raza, religión, ideología o un simple rechazo por una preferencia, son también signos de violencia, violencia escolar. Lo que sucede con estos actos es que se han convertido en sucesos normales, en cosas que pasan, que no interesan, que no generan preocupación. No resulta alarmante lo que está pasando dentro de las escuelas, o más bien, lo que no está pasando: una educación en sana convivencia, en habilidades humanas y desarrollo humano que contribuya a la construcción de paz.

Siguiendo la teoría de Johan Galtung (2009) es posible que el conflicto esté latente en las sociedades, lo que no es constante es que se manifieste a través de la violencia o la guerra y es en ese punto en el que hay que trabajar. Para entender cómo se manifiesta la violencia existe una clasificación de esta: la violencia directa; es aquella que es visible, por tanto, es la violencia que resulta fácil de detectar y posiblemente de combatir. Está en un escenario notorio que comprende acciones agresivas contra personas, colectividades, o también a la naturaleza. Por tanto, se manifiesta de manera física, verbal o psicológica. Galtung (s.f.b).

También hace mención de otros tipos de violencia: la estructural y la cultural que tienen causas más profundas y no tan visibles como ocurre en la violencia directa. Al hablar de no visibles no se quiere decir que no sean notorias, sino que no son perceptibles por medio de la vista. En la primera de estas se produce una afectación a la satisfacción de necesidades humanas, pero que no es tan fácil de identificar, no hay un agente como tal que realice actos notorios de violencia, sino que esta está inmersa en la estructura social, por ejemplo, casos en los que el sistema causa hambre, enfermedad o cualquier afectación de tal tipo a la población; tiene que ver con la desigualdad y la injusticia. Galtung (s.f.b).

En cuanto a la violencia cultural, tiene relación con aspectos de la cultura que se expresan a través de distintos medios como la religión, el lenguaje, el arte, la ciencia, entre otros, que propenden por justificar alguno de los otros tipos de violencia. 
Estos tipos de violencia no están aislados ni tampoco son excluyentes, por el contrario, suelen evidenciarse simultáneamente. En relación con la construcción de paz, habría que evitar cualquier tipo de violencia antes de que aparezca, o tratar de mitigarla una vez se presente. Menciona García (s.f) que:

Las estructuras violentas no se pueden solucionar mediante la violencia, pues ello llevaría a esta misma estructura y, además, reforzaría una cultura bélica. La forma de romper ese círculo vicioso es anteponer una estructura y una cultura de paz donde existan los mecanismos necesarios para solventar los conflictos por medios no violentos. (p.131).

Más allá de formular soluciones que acaben el conflicto, nuestro interés es crear propuestas que trasciendan en él, lo transformen, que logren dirimir diferencias que generan incompatibilidad en la sociedad y que impiden una convivencia pacífica, empezando por la escuela, lugar base del desarrollo humano, del ciudadano propositivo con habilidades humanas constructivas.

\section{Paz}

Analicemos la definición de Paz que propone Galtung: "ausencia de violencia directa, estructural y cultural más la presencia de mecanismos para la resolución de conflictos" (Galtung, 2009, p.131). De allí surge la idea de la conexión que existe entre el proyecto de atención a estudiantes víctimas de matoneo por razones de orientación sexual que adelantamos como línea de género y derecho de la clínica jurídica y el proceso de paz que atraviesa Colombia. ¿Por qué? porque la violencia escolar se enmarca dentro de los tres tipos de violencia que describe Johan Galtung en su teoría de conflictos.

Según la Constitución política de Colombia: "La Paz es un derecho y un deber de obligatorio cumplimiento" (Const., 1991, art. 22). En la actualidad adelantamos un proceso de paz en Colombia y es importante ver este postulado como un derecho fundamental de nuestra carta magna, teniendo el compromiso como ciudadanos de ver la Paz no solo como un derecho sino también como un deber que se ejerce en el día a día y participando de manera más activa en la política ciudadana, no solo para hacer veeduría del proceso, sino aportar desde los diferentes ámbitos de la sociedad, entre ellos las instituciones escolares: el ámbito educativo. Apareciendo el siguiente artículo de nuestra Constitución Política de 1991:

Son derechos fundamentales de los niños: la vida, la integridad física, la salud y la seguridad social, la alimentación equilibrada, su nombre y nacionalidad, tener una familia y no ser separados de ella, el cuidado y amor, la educación y la cultura, la recreación y la libre expresión de su opinión. Serán protegidos contra toda forma de abandono, violencia 
física o moral, secuestro, venta, abuso sexual, explotación laboral o económica y trabajos riesgosos. Gozarán también de los demás derechos consagrados en la Constitución, en las leyes y en los tratados internacionales ratificados por Colombia. La familia, la sociedad y el Estado tienen la obligación de asistir y proteger al niño para garantizar su desarrollo armónico e integral y el ejercicio pleno de sus derechos. Cualquier persona puede exigir de la autoridad competente su cumplimiento y la sanción de los infractores. Los derechos de los niños prevalecen sobre los derechos de los demás. (Const., 1991, art. 44).

Podemos examinar la última parte del párrafo en donde dice de manera expresa que los derechos de los niños y niñas prevalecen sobre los demás derechos, y precisamente es esto lo que nos lleva a pensar que no podemos olvidarlos en este proceso. Los niños y niñas tienen derecho a la paz: a desarrollarse en un ambiente constructivo donde ellos también proponen, participan, entienden su mundo y generan cambios.

\section{Análisis desde la perspectiva de los derechos humanos y la ley}

Es importante tener en cuenta el artículo primero de la Declaración Universal de Derechos Humanos Adoptada y proclamada por la Asamblea General en su resolución 217 A (III), de 10 de diciembre de 1948 que dice: "Todos los seres humanos nacen libres e iguales en dignidad y derechos y, dotados como están de razón y conciencia, deben comportarse fraternalmente los unos con los otros." (Art. 1).

Entendemos que los derechos humanos son atributos inherentes a todas las personas sin discriminación por razón de tipo personal, social o cultural. Y su aplicación corresponde al Estado y a los individuos que hacen parte de las diferentes relaciones sociales, es la característica que los hace esenciales en la práctica para la no violencia en una sociedad. Este postulado nos da una idea de derechos humanos que tienen como base la igualdad y la libertad en dignidad. Sin embargo, para muchas personas, lamentablemente, esto no se cumple, pues sus derechos humanos son transgredidos, y es en este punto donde los sistemas, órganos, instrumentos normativos y estándares de aplicación jurisprudencial y procedimental de derechos humanos se ponen en marcha para garantizar y proteger los derechos humanos de todos los individuos que invoquen amparo frente a las cortes internacionales. Es necesario el cumplimiento de los derechos humanos para el cambio social en la construcción de paz. Por esta razón somos todos responsables de materializarlos, tanto el estado como los ciudadanos, comportándonos, como lo dice la Declaración, fraternalmente los unos con los otros, y enseñando estos fundamentos en las instituciones educativas, pues son el primer lugar en donde, como individuos, tenemos relaciones en el marco de la ciudadanía. Como bien lo menciona el prefacio de la Declaración Universal de Derechos humanos: 
Proclama la presente Declaración Universal de Derechos Humanos como ideal común por el que todos los pueblos y naciones deben esforzarse, a fin de que tanto los individuos como las instituciones, inspirándose constantemente en ella, promuevan, mediante la enseñanza y la educación, el respeto a estos derechos y libertades, y aseguren, por medidas progresivas de carácter nacional e internacional, su reconocimiento y aplicación universales y efectivos, tanto entre los pueblos de los Estados Miembros como entre los de los territorios colocados bajo su jurisdicción. (p.1)

Para que los derechos humanos dejen de ser un ideal inalcanzable y se materialicen con plenitud necesitamos promoverlos mediante la educación y promoción.

La Ley 1620 de 2013 tuvo como objetivo central la creación del sistema nacional de Convivencia escolar con miras a prevenir la violencia escolar y también a mitigarla. Esta ley no ha sido materializada, no se han creado los comités o no se han consolidado, puesto que la línea que habría de seguir esta normatividad estaba basada en la modificación de los manuales de convivencia según pautas que la misma ley aportaba; no obstante, ha sido un tema puesto en la lista de pendientes sin ejecutar.

Pese a que no ha sido implementada plenamente la ley 1620 de 2013 y las ordenes de la Sentencia T-478/2015 ${ }^{4}$, han dado al ámbito escolar unos lineamientos importantes en lo que respecta a la convivencia. Así, el artículo 11 además de explicar las funciones que ha de cumplir el comité, hace mención del hecho de articular políticas, estrategias y programas teniendo relación con el desarrollo de la ciudadanía. De los derechos humanos y también de los derechos sexuales y reproductivos.

Con estos elementos de contexto es necesario articular esfuerzos para generar alternativas para mitigar la violencia en la escuela y construir escenarios de paz.

\section{PROPUESTA: LA ESCUELA COMO UN TERRITORIO DE PAZ}

Como un ejercicio de promoción de la convivencia escolar como aporte a la construcción de paz en Colombia, se propone un ejercicio a través de la narración oral del cuento: "El Puente hacia la Paz", de la escritora Paola Esteban (2017). El texto tiene como temática principal la resolución de conflictos y los derechos a la diversidad y a la paz con el fin de impactar a las nuevas generaciones de agentes de paz: las niñas y niños colombianos. El texto se adapta para trabajar con los estudiantes de primaria y la actividad que implementaremos tiene como finalidad: prevenir y mitigar

4 El caso de Sergio Urrego puso en evidencia este hecho, así es reconocido por la Corte en la Sentencia T-478 de 2015 
el matoneo en las instituciones escolares como reflejo de la consolidación de paz en las diferentes esferas sociales, entre ellas la escuela. Así mismo, tenemos el propósito de impartir a los estudiantes nociones básicas de derechos humanos y derechos fundamentales constitucionales como herramientas útiles para la paz escolar y resolución de conflictos. Siendo el texto "el Puente hacia la Paz" coherente con nuestros objetivos.

\section{Justificación}

Como Clínica Jurídica de Derecho e Interés Público y como línea de género y diversidad, unificaremos la tecnicidad del Derecho con el lenguaje artístico para así obtener diversas y positivas reflexiones por parte de los niños y niñas de las escuelas a las que podamos llegar y fomentar los derechos humanos que serán útiles para mejorar la Convivencia Escolar, y también las relaciones en las diferentes áreas de los niños y niñas de los primeros grados. El objetivo es impartir en las escuelas nociones de derechos humanos y derechos constitucionales, teniendo en cuenta que estos postulados no son únicos de la esfera de los juristas o académicos, sino que son patrimonio de toda la humanidad.

Es importante resaltar que en la construcción de paz en las escuelas que nos proponemos en este proyecto se contará con la activa participación del cuerpo docente de las instituciones, teniendo en cuenta que ellos son parte de este proceso y son quienes seguirán acompañando a los estudiantes en su formación ciudadana.

\section{Objetivos}

- Analizar la violencia en las escuelas y, junto a los estudiantes, crear propuestas para disminuir las situaciones que atente contra la paz escolar.

- Dar a conocer a los niños, niñas y adolescentes los derechos humanos como base de la convivencia escolar.

- Promover el arte como lenguaje en la construcción de paz desde la Escuela.

- Establecer y crear unos principios generales en los diferentes grupos de niños y niñas de las escuelas y acordar su aplicación para la mejora de las relaciones entre ellos y en la sociedad como aporte a la construcción de paz en Colombia.

Con los estudiantes de básica primaria se expondrá el cuento por medio de narración oral, de esta manera, los estudiantes podrán apropiarse de la construcción de paz en una comunidad para luego proponer como construir paz en su entorno escolar. Posterior a la lectura habrá una exposición de lo que son los derechos humanos universales, cuáles son y cómo se deben desarrollar en la vida cotidiana. También hablaremos del concepto de 
paz global y escolar. La actividad finaliza con la elaboración de un dibujo por parte de cada estudiante que evidencie cómo y con qué identifican la paz en sus respectivos salones de clase.

Con los estudiantes de básica secundaria también se hará la narración oral del cuento que hemos trabajado y se impartirán nociones básicas de derechos humanos, paz escolar y convivencia. Cada estudiante deberá promover un principio que empiece a regir en sus relaciones, en su institución escolar y que aporte a la construcción de paz.

Como estudiantes de clínica nos proponemos adelantar una campaña con el fin de educar en materia de derechos humanos a las comunidades escolares. La idea es visitar las instituciones y/o convocar a los estudiantes a seminarios en materia de derechos humanos y estrategias que pueden impartir en la escuela para mitigar la violencia. Estos seminarios se dictarán en la Universidad Autónoma de Bucaramanga. También nos proponemos adelantar acciones ciudadanas para seguir de cerca el proceso que lleva la ley 1620 de 2013 en las escuelas. Además, revisar cuáles de las órdenes de la sentencia T-478 de 2015 se han cumplido en las instituciones escolares de Bucaramanga y su área metropolitana: la justicia ha dado respuesta a esta problemática, pero es necesario que se ponga en marcha lo que se ha promulgado mediante la ley; por eso creemos que el rol de la sociedad civil a través de los movimientos sociales es fundamental para el cambio social, el desarrollo y la aplicación de políticas públicas. Por tanto, como estudiantes de la Clínica Jurídica y su relación sociedad-acción, fomentamos el activismo legal y cooperamos con los movimientos sociales.

En Entrevista realizada por El Espectador, la psicóloga Florencia Brandoni, menciona lo siguiente:

Gran parte de la conflictividad que se ve en los colegios tiene que ver con la necesidad de construir identidad. Es entonces cuando empiezan a adquirir una gran relevancia el grupo de pares y la formación de pequeños grupos suele volverse crucial, como una batalla cotidiana. El juego de las diferencias se da con cualquier elemento, no solo el estrato socioeconómico: quienes son rubios, altos, gordos, los más deportistas o los estudiosos. Ese juego de diferenciaciones en ocasiones se vuelve muy cruel. Padecer la violencia de los compañeros está muy ligado a la falta de integración grupal, en eso puede jugar cualquiera de las diferencias mencionadas y la falta de recursos puede crear una vulnerabilidad extra. (El espectador, 28 de septiembre de 2016).

Hay que encontrar salidas eficaces para los problemas que existen en las instituciones escolares. Las instituciones deben adecuar sus dinámicas de enseñanza para las víctimas del conflicto armado, del microtráfico, del reclutamiento, las víctimas de acoso escolar. La escuela debe ser un espacio en donde se promueva y se garantice el cumplimiento de los derechos humanos. 


\section{CONCLUSIONES}

Como Clínica Jurídica de Interés Público y Derechos Humanos creemos que no basta con educar o trabajar solamente con los agentes directos del conflicto, es decir, con víctimas, ex-paramilitares, reincorporados a la sociedad, ex-guerrilleros y militares.

De manera colectiva somos responsables del fin del conflicto armado en Colombia, por lo tanto, necesitamos, en palabras de Lederach (2000), un cambio social colectivo puesto que

La construcción de la paz exige una visión de la relación. Dicho sin rodeos, si no hay capacidad para imaginarse el lienzo de las relaciones mutuas y de situarse a sí mismo como parte de esa red histórica y constante evolución la construcción de la paz se viene abajo. (p.132).

La importancia de la participación ciudadana mediante acciones legales populares, y el seguimiento a las instituciones del estado, así como el activismo por parte de la sociedad civil como herramienta para la transformación social y mediante reformas de carácter legal, son procesos que, como estudiantes de la clínica jurídica, no podemos perder de vista para el alcance de nuestros objetivos.

Los niños, niñas y jóvenes colombianos deben conocer y ser parte del proceso de paz en Colombia. Para ello deben vivir en una atmósfera escolar donde los conflictos se resuelvan de forma pacífica y concertada, donde además la diferencia sea respetada.

La violencia en las escuelas es una realidad que no se puede negar y que genera trágicas consecuencias. La escuela es el lugar en donde niños y niñas se relacionan como futuros ciudadanos agentes de derechos y de cambio. Pero no podrán hacerlo si desconocen sus derechos humanos y si no tienen garantías por parte del Estado para la protección de estos derechos fundamentales.

La paz es más que una conclusión abstracta a la que se quiere llegar con el fin del conflicto armado: la paz es una construcción formada en la vida cotidiana, en las calles, en el comercio, en el trabajo, en casa, y también en las instituciones escolares.

\section{REFERENCIAS}

Botero, D. (2005). Derecho a la Utopía. Bogotá: Universidad Nacional de Colombia.

Constitución política de Colombia (1991) 35a Ed. Legis

Congreso de Colombia (noviembre 8 de 2006) Código de la Infancia y la Adolescencia. [Ley 1098 de 2006] DO: 46446 
¿Cómo hacer la paz desde los colegios? El Espectador, 28 de septiembre de 2016. http://colombia2020.elespectador.com/pais/como-hacerla-paz-desde-los-colegios

D’Angelo, L., \& Fernández, D. UNICEF. (2011). Clima, conflictos y violencia en las escuelas. Recuperado de https://www.unicef.org/argentina/spanish/clima_conflicto_violencia_escuelas.pdf

Esteban, P. (2017). El puente hacia la paz. Recuperado de: http://megustaescribir.com/obra/leer/60593/el-puente-hacia-la-paz

Galtung, J. (2009a) P. Calderón Teoría de conflictos de Johan Galtung. Revista paz y conflictos, 2. 60 - 81. Recuperado de http://www.redcimas.org/wordpress/wp-content/uploads/2012/08/m_JGaltung LAteoria.pdf

Galtung, J. (s.f.b) García, V. La transformación de los conflictos por medios pacíficos. Recuperado de https://dialnet.unirioja.es/descarga/ articulo/595158.pdf

Lederach, J. (2000). La imaginación moral, El arte y el alma de construir la paz. Barcelona, España. Grupo editorial Norma.

Vásquez, J. (2008). Fundamentos para la creación de una clínica jurídica en la FUNLAM como apoyo en la enseñanza práctica del derecho. Revista IIEC, 2(3), 11- 21.

Vásquez, J., \& Correa, L. (2008). La enseñanza clínica del Derecho: transformando la forma de enseñar y ejercer el Derecho. Studiositas, $3(1), 34-40$. 